\title{
Sp1 and Sp3 regulate glucokinase gene transcription in the liver of gilthead sea bream (Sparus aurata)
}

\author{
M Egea, I Metón and I V Baanante \\ Departament de Bioquímica i Biologia Molecular, Facultat de Farmàcia, Universitat de Barcelona, Diagonal 643, 08028 Barcelona, Spain \\ (Requests for offprints should be addressed to I V Baanante; Email: baanantevazquez@ub.edu)
}

\begin{abstract}
To better understand the transcriptional machinery that governs glucokinase (GCK) expression, we have cloned and characterized the proximal promoter region of GCK from gilthead sea bream (Sparus aurata). The $5^{\prime}$-flanking region of GCK was isolated by chromosome walking. SMART RACE-PCR allowed us to locate the transcription start site $98 \mathrm{bp}$ (bp) upstream from the translational start. Transfection analysis in HepG2 cells revealed the presence of a functional promoter in the $1397 \mathrm{bp} 5^{\prime}$-flanking isolated fragment (positions -1321 to +76 relative to the transcription start site). Sequential $5^{\prime}$-deletion analysis indicated a core functional promoter for basal transcription within the 288 bp upstream from the transcription start site. Transient transfection experiments performed in HepG2 cells and electrophoretic mobility shift assays denoted that Sp1 binds and transactivates GCK promoter, whereas Sp3 repressed Sp1-mediated activation of GCK by competing for the same binding site. Mutations in the Sp binding site completely abolished the enhancing effect of Sp1. Treatment with insulin stimulated GCK expression, and increased Sp1 levels in S. aurata liver. We propose a new mechanism that involves Sp1 and Sp3 to mediate insulin activation of GCK transcription.
\end{abstract}

Journal of Molecular Endocrinology (2007) 38, 481-492

\section{Introduction}

Glucokinase (GCK, ATP: D-hexose 6-phosphotransferase, EC 2.7.1.1), or hexokinase IV, catalyzes the phosphorylation of glucose to glucose-6-phosphate (Glu-6$\mathrm{P})$. In mammals, GCK is mainly expressed in liver and pancreatic $\beta$-cells. The hepatic enzyme contributes to the control of glycemia through glucose metabolization and its storage as glycogen, whereas the pancreatic isoform acts as a glucose sensor that controls insulin secretion in $\beta$-cells. In mammals, starvation and diabetes downregulate $G C K$ expression in the liver, whereas refeeding on high carbohydrate diets and treatment with insulin stimulate GCKexpression. Glucocorticoids potentiate the action of insulin, whereas 3,5,3'-triiodo-L-thyronine and biotin also stimulate $G C K$ transcription, but to a lesser extent than insulin (Iynedjian et al. 1989, Magnuson et al. 1989, Magnuson 1990, Narkewicz et al. 1990, Chauhan \& Dakshinamurti 1991, Iynedjian 1993). The stimulatory effect of insulin on hepatic GCK expression is reversed by cAMP (Iynedjian et al. 1989). The mammalian GCK gene has a dual promoter transcription unit. The upstream promoter is active in pancreatic islets $\beta$-cells, duodenum, pituitary, and brain, whereas the downstream promoter is active in hepatocytes (Magnuson \& Shelton 1989, Iynedjian et al. 1996). The mechanism involved in liverspecific expression of $G C K$ is not well understood. Hypoxia-inducible factor-1 (Roth et al. 2004), sterolregulatory element-binding protein-1 (SREBP-1; Foretz et al. 1999), signal transducer and activator of transcription 5B (STAT 5B; Sawka-Verhelle et al. 2000), upstream stimulatory factors (USFs; Iynedjian 1998), hepatocyte nuclear factor (HNF)-4 (Roth et al. 2002), and the transcriptional coactivator p300 (Roth et al. 2004) have been reported as insulin-dependent $G C K$ gene activators. A recent report challenges involvement of STAT 5B in GCK transcription (Ribaux et al. 2002). Whether SREBP-1 mediates insulin action on GCK expression is also controversial (Gregori et al. 2006, Hansmannel et al. 2006). Other HNFs, FoxO1, and peroxisome proliferatoractivated receptor- $\gamma$ have also been proposed to play a role in GCK transcription in the liver (Cha et al. 2001, Lannoy et al. 2002, Kim et al. 2004, Zhang et al. 2006).

In fish, glucose intolerance and limited use of dietary carbohydrates have been reported. Compared with mammals, these animals show prolonged hyperglycemia after a glucose load and when feeding on high carbohydrate diets (Christiansen \& Klungsøyr 1987, Cowey \& Walton 1989, Wilson 1994, Moon 2001, Metón et al. 2003). The relative inability of carnivorous fish to utilize carbohydrates has been reported as being due, in part, to absence of GCK activity in the liver (Wilson 1994, Moon 2001). Recently, the cDNA coding for GCK has been isolated from the piscine liver and the functionality of the fish GCK CDNA was shown by in vitro transcription/translation and after transient transfection in COS-7 cells (Caseras et al. 2000). These findings indicate that GCK is functional in fish liver, 
although control of $G C K$ gene expression remains poorly understood.

To gain insight into the molecular mechanism regulating $G C K$ expression, we have cloned and characterized the proximal promoter region of $G C K$ from gilthead sea bream (Sparus aurata). We conclude that Sp1 transactivation of GCK promoter and repression of Sp1-mediated activation by Sp3 may be involved in insulin action on $G C K$ expression.

\section{Materials and methods}

\section{Animal treatments}

Gilthead sea bream (S. aurata) obtained from Tinamenor (Cantabria, Spain) were maintained as described previously (Salgado et al. 2004). Fish were fed daily $(1000 \mathrm{~h})$ at $1 \%$ body weight. To study the regulation of GCK, Sp1, and Sp3 expression by insulin in vivo, $24 \mathrm{~h}$ after the last meal $(1000 \mathrm{~h})$, two different groups received an i.p. injection of bovine insulin ( $10 \mathrm{U} / \mathrm{kg}$ fish; Sigma) or vehicle (saline). Fish were killed by cervical section $6 \mathrm{~h}$ after treatment. Tissue samples were dissected out, immediately frozen in liquid $\mathrm{N}_{2}$ and kept at $-80^{\circ} \mathrm{C}$ until use. To avoid stress, fish were anesthetized with MS-222 (1:12 500) before handling. The experimental procedures met the guidelines of the animal use committee of the Universitat de Barcelona.

\section{Cloning of the $5^{\prime}$-flanking region of GCK gene from $S$. aurata by chromosome walking}

The 5'-flanking region of GCK was isolated by PCR using the Universal GenomeWalker Kit (Clontech). Briefly, four libraries were obtained by blunt-end digestion of $S$. aurata genomic DNA with DraI, EcoRV, PvuII, and StuI. Each library was ligated to the GenomeWalker adaptor provided in the kit. A primary PCR was performed on each library with the gene-specific primer ME0001 (5'-CCGCAGGTTT GACTAAATGAGCC-3' ${ }^{\prime}$ and the AP-1 primer provided in the kit. A nested PCR was performed on the primary PCR product using the gene-specific primer ME0004 (5'-GCCTCGGTATGTGTCTCTAGGTGTGTGG-3') and the AP2 primer from the kit. The longer amplification product, a single $1.4 \mathrm{~kb}$ band, was obtained from the StuI library and ligated into pGEM T easy plasmid (Promega) to generate pGEM-GK1400. Two independent clones were fully sequenced on both strands following the ABI Prism BigDye Terminator Cycle Sequencing Ready Reaction kit instructions (Applied Biosystems, Foster City, CA, USA).

\section{Characterization of the transcription start site}

The $5^{\prime}$ end of the hepatic $S$. aurata GCK cDNA was determined using the SMART RACE cDNA
Amplification kit (Clontech). One microgram poly $\mathrm{A}^{+}$RNA obtained from liver of $S$. aurata was converted into cDNA using PowerScript reverse transcriptase (RT) at $42{ }^{\circ} \mathrm{C}$ for $1.5 \mathrm{~h}$ in the presence of $5^{\prime}$-CDS primer, for first-strand synthesis, and SMART II A oligonucleotide, which serves as an extended template for RT after annealing an oligo$\mathrm{dC}$ previously added to the end of the mRNA template by the terminal transferase activity of PowerScript RT. Primary and nested PCR were conducted with primer pairs ME0001/Universal Primer Mix A (from the kit) and ME0004/Nested Universal Primer (from the kit) respectively. The single $106 \mathrm{bp}$ band generated was purified and ligated into pGEM $\mathrm{T}$ easy plasmid (Promega). Identical nucleotide sequence corresponding to the $5^{\prime}$ end of GCK cDNA was obtained by sequence analysis of two independent clones.

\section{RT-PCR analysis of GCK mRNA tissue specificity}

cDNA templates for PCR amplification were synthesized from $5 \mu \mathrm{g}$ total RNA obtained from $S$. auratafrozen tissues using Moloney Murine Leukemia Virus (M-MLV) RT (BRL Life Technologies). The RT reaction products were subjected to PCR amplification using MEGK01 (5'-CCGTGTGATGCTGGTGAAGG-3') and MEGK02 $\left(5^{\prime}\right.$-GGAGAAGGTGAAACCGAGAGG-3 $\left.{ }^{\prime}\right)$ as primers to amplify a $202 \mathrm{bp}$ fragment of $S$. aurata GCK cDNA. As a control of RNA integrity, a $711 \mathrm{bp}$ fragment of $S$. aurata $\beta$-actin was amplified in parallel with primers BA0199 (5'-GACAACGGATCCGGTATGTGC- $3^{\prime}$ ) and BA0299 $\left(5^{\prime}\right.$-GACCTGTCCGTCGGGCAGCTC- $\left.{ }^{\prime}\right)$. Aliquots of each reaction were electrophoresed on a $1 \%$ agarose gel and visualized by ethidium bromide staining.

\section{Construction of reporter gene plasmids}

The $S$. aurata GCK promoter sequence located between positions -606 to +76 relative to the transcription start site was generated by PCR, using pGEM-GK1400 as template and oligonucleotides ME0107 (5'CTCCTAAATTCAGACCCCAC-3' ${ }^{\prime}$ ) and ME0004, and subcloned into the SmaI site of the promoterless luciferase reporter plasmid pGL3-Basic (Promega) to generate pGK606. The pGK1321 construct was made by ligation of the NcoI/NdeI GCK promoter fragment, obtained from pGEM-GK1400, to the MluI/NdeI site of pGK606. To this end, NcoI and MluI ends were made blunt before NdeI digestion and subsequent ligation. The reporter constructs designated as pGK288 and pGP21 were produced by self-ligation of filled-in ends of pGK606 after digestion with EcoRV/NheI and PvuII/NheI respectively. The pGK288mutSp construct was made by ligation of the MluI/PvuII fragment of a PCR product, obtained 
using pGK288 as template and oligonucleotides ME0506 (5'-GAGAACTTCAGCTGTTTTTATGCTCA GGTGAAGTTTATTTTATACACCGCACGTAAAGGC

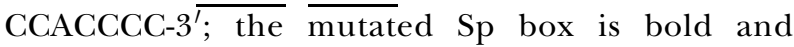
double-underlined) and RVprimer3 (positions +4760 to +4779 in pGL3-Basic vector), into pGK288 previously digested with MluI/PvuII. The pGK72 and pGK72mutSp constructs were generated by PCR amplification using pGK606 as template, and primer pairs ME0202 (5'-CCCCCGGGCCTTTACGTGCGG-3'; the SmaI site included is bold and underlined)/GL primer2 (positions +111 to +89 in pGL3-Basic vector) and ME0310 (5'-CCCCCGGGCCTTTACGTGCGGTG TATAAAATAAACTTCACCTGAGCATAAAAACAG-3'; the SmaI site included is bold and underlined; the mutated Sp box is bold and double-underlined)/ GLprimer2 respectively. The PCR products were subcloned into the SmaI/HindIII site of pGL3-Basic. Self-ligation of pGK606 previously digested with EcoRV/PvuII was performed to generate pGK606 288 -21. The reporter construct harboring the rat liver GCK promoter (prGK1418) was constructed by ligation of a PCR product, obtained by amplification using oligonucleotides ME0503 (5'-GCTCCCTCCTCTCCGGAGGC-3') and ME0504 ( $5^{\prime}$-GGGGTCGTTGGGGAAGTAGAG-3 ${ }^{\prime}$ ) and genomic DNA from rat as template, into the SmaI site of pGL3-Basic. All constructs were verified by sequencing using ABI Prism BigDye Terminator Cycle Sequencing Ready Reaction kit (Applied Biosystems).

\section{Cell transfection and luciferase assay}

HepG2 (ATCC HB 8065) were cultured in Dulbecco's modified Eagle's medium (D-MEM), supplemented with $10 \%$ fetal bovine serum, $100 \mathrm{IU} / \mathrm{ml}$ penicillin, $100 \mu \mathrm{g} / \mathrm{ml}$ streptomycin, and $2 \mathrm{mM}$ glutamine. The calcium phosphate co-precipitation method was used for transient transfection of HepG2 at 45-50\% confluence in six-well plates (Graham \& Van der Eb 1973). Cells were transfected with $4 \mu \mathrm{g}$ reporter construct and with $400 \mathrm{ng}$ expression vectors encoding Sp1 or Sp3. To correct for variations in transfection efficiency, $500 \mathrm{ng}$ CMV- $\beta$ (lacZ) was included in each transfection. To ensure equal DNA amounts, empty plasmids were added in each transfection. Four hours after addition of the precipitate, cells were shocked in $10 \%$ dimethylsulfoxide in serum-free medium for $2 \mathrm{~min}$. The cells were harvested $16 \mathrm{~h}$ later, washed in PBS and incubated for $15 \mathrm{~min}$ in $300 \mu$ l Cell Culture Lysis Reagent (Promega). After the removal of cell debris by centrifugation at $10000 \mathrm{~g}$ for $15 \mathrm{~s}$, luciferase activity was measured in the supernatant after the addition of Luciferase Assay Reagent (Promega) in a TD-20/20 Luminometer (Turner Designs, Sunnyvale,
CA, USA). $\beta$-Galactosidase activity of 30-100 $\mu \mathrm{l}$ clear lysate was measured in a $1 \mathrm{ml}$ reaction containing $0.3 \mathrm{mM} \mathrm{MgCl}_{2}, 13.5 \mathrm{mM} \beta$-mercaptoethanol, $0.9 \mathrm{mM}$ 2-nitrophenyl- $\beta$-D-galactopyranoside (ONPG), and $0 \cdot 1 \mathrm{M}$ sodium phosphate $\mathrm{pH} 7 \cdot 5$. After incubation at $37^{\circ} \mathrm{C}$, the reaction was stopped by addition of $0.5 \mathrm{ml}$ of $0.5 \mathrm{M} \mathrm{Na}_{2} \mathrm{CO}_{3}$, and the intensity of the yellow color was determined by its optical density at $420 \mathrm{~nm}$. Culture medium for Chinese hamster ovary $(\mathrm{CHO})$ cells was Ham's F-12 nutrient mixture supplemented with $10 \%$ fetal bovine serum, $100 \mathrm{IU} / \mathrm{ml}$ penicillin, $100 \mu \mathrm{g} / \mathrm{ml}$ streptomycin, and $2 \mathrm{mM}$ glutamine. Sea bass larvae (SBL) cells were cultured at $20^{\circ} \mathrm{C}$ in MEM, complemented with Hank's salts, $10 \%$ fetal bovine serum, $100 \mathrm{IU} / \mathrm{ml}$ penicillin, $100 \mu \mathrm{g} / \mathrm{ml}$ streptomycin, and $2 \mathrm{mM}$ glutamine. CHO and SBL cells were transiently transfected with reporter and expression constructs using FuGene 6 (Roche), according to the manufacturer's instructions. As an internal control, CMV- $\beta$ was included in each transfection. Cells were harvested $40 \mathrm{~h}$ following transfection, and luciferase, and $\beta$-galactosidase activity were assayed as for HepG2 cells. The SBL cell line was a generous gift of Dr J Castric (Technopôle Brest-Iroise, Brest, France). Expression plasmids encoding rat Sp1 (pRC-CMVSp1) and rat Sp3 (pRC-CMVSp3) were kindly provided by Dr G Suske (Institute of Molecular Biology and Tumor Research, Marburg, Germany; Hagen et al. 1994).

\section{Electrophoretic mobility shift assay}

Double-stranded oligonucleotides used in gel shift experiments were (only the forward oligonucleotides are shown): Spl-cons (5'-AAGAGGGTGGGCGGGAG CAACCA-3'), GK-56/-37 (5'-TGTGGCCAGCCCACTTC ACC- $\left.-3^{\prime}\right)$, and GK-56/-37mutSp ( $5^{\prime}$-TGTATAAAATAAAC TTCACC-3 ${ }^{\prime}$ ). Double-stranded oligonucleotides, 100 pmols, were 3'-end labeled with digoxigenin11-ddUTP using terminal transferase (Roche) in a $20 \mu \mathrm{l}$ reaction for $30 \mathrm{~min}$ at $37^{\circ} \mathrm{C}$. The reaction was stopped by adding $2 \mu \mathrm{l} 0 \cdot 2 \mathrm{M}$ EDTA. Binding reactions were carried out in a total volume of $20 \mu \mathrm{l}$ containing $100 \mathrm{mM}$ Hepes, pH 7•6, $5 \mathrm{mM}$ EDTA, $50 \mathrm{mM}\left(\mathrm{NH}_{4}\right)_{2} \mathrm{SO}_{4}$, $5 \mathrm{mM}$ dithiothreitol, $1 \%$ Tween $20,150 \mathrm{mM} \mathrm{KCl}, 1 \mu \mathrm{g}$ non-specific competitor poly [d(I-C)] (Roche), HepG2 extracts prepared from Sp1 or Sp3 overexpressing cells or in vitro translated $\mathrm{Sp} 1$ and $\mathrm{Sp} 3$ proteins, and the labeled probe. In vitro transcription/translation of Sp1 and Sp3 proteins were made with TNT T7 quick coupled transcription/translation system (Promega) in a reaction mixture incubated for $1 \mathrm{~h}$ at $4^{\circ} \mathrm{C}$. The DNA-protein complexes were electrophoresed at $4{ }^{\circ} \mathrm{C}$ on $5 \%$ polyacrylamide gel and using $0.5 \times$ tris-borate-EDTA (TBE) as buffer. DNA was thereafter transferred by contact blotting ( $2 \mathrm{~h}$ at room temperature) to Nytran membranes (Schleicher \& Schuell, Keene, NH, USA). 
DNA was cross-linked to membranes by UV irradiation for 3 min. Labeled probes were immunodetected with anti-digoxigenin conjugated to alkaline phosphatase (Roche) and using CDP-Star (Roche) as chemiluminescent substrate. Membranes were finally exposed to Hyperfilm ECL (Amersham). For competition experiments, HepG2 extracts were preincubated for $30 \mathrm{~min}$ with a 10- to 200-fold molar excess of unlabeled double-stranded Sp1-cons oligonucleotide. For supershift assays, $2 \mu \mathrm{g}$ polyclonal anti-human Sp1 and/or Sp3 (Santa Cruz Biotechnology, Santa Cruz, CA, USA) were incubated with in vitro translated Sp1 and Sp3 proteins for $30 \mathrm{~min}$ at room temperature before addition of the probe.

\section{Northern blotting analysis}

Total RNA was isolated from liver of $S$. aurata with the Total Quick RNA Cells \& Tissues Kit (Talent, Trieste, Italy), electrophoresed on a $1 \%$ agarose-formaldehyde gel, transferred to a nylon membrane, and crosslinked to the membrane by UV irradiation. A GCK homologous probe was labeled by incorporation of digoxigenin-11-dUTP during PCR with primer pair MEGK01/MEGK02. Prehybridizations of the membranes were performed at $50{ }^{\circ} \mathrm{C}$ for $2 \mathrm{~h}$ in $7 \%$ SDS (wt/vol), $50 \%$ formamide, $5 \times$ SSC, $2 \%$ blocking reagent (Roche), $0 \cdot 1 \%$ laurylsarcosine (wt/vol), $50 \mathrm{mM}$ sodium phosphate $(\mathrm{pH} \mathrm{7 \cdot 0)}$. The probe was added and hybridization was carried out overnight at $50{ }^{\circ} \mathrm{C}$. High stringency washes were performed as described (Salgado et al. 2004). The 18S rRNA was used to correct for loading irregularities. Labeled probes were immunodetected with anti-digoxigenin conjugated to alkaline phosphatase and CDP-Star as described above.

\section{Western blotting analysis}

Proteins in liver crude extracts from $S$. aurata were separated on $10 \%$ SDS-PAGE gels, transferred to nylon membranes and immunoblotted with polyclonal anti human Sp1 and Sp3 (Santa Cruz Biotechnology) following the ECL Western blotting (Amersham) procedure. An anti-rabbit peroxidase-conjugated secondary antibody was used for chemiluminescent detection.

\section{Results}

\section{Cloning of the $5^{\prime}$-flanking region of the S. aurata GCK gene}

A $1397 \mathrm{bp}$ fragment upstream from the translation start codon of $G C K$ was isolated by chromosome walking on $S$. aurata genomic DNA using oligonucleotides designed from the piscine hepatic GCK cDNA (Fig. 1). GCK expression was assessed by RT-PCR in various tissues of $S$. aurata. In piscine tissues, expression of GCK mRNA was essentially found in the liver. The putative transcription initiation site of GCK mRNA was determined using the SMART RACE PCR approach (Fig. 2A). A single fragment was obtained and cloned into pGEM $\mathrm{T}$ easy. Upon sequencing, this fragment exhibited complete homology to the 47 bases in the $5^{\prime}$ untranslated region of the previously cloned $S$. aurata GCK cDNA (Caseras et al. 2000). Analysis of two independent clones indicated that $S$. aurata GCK mRNA initiates 98 nucleotides upstream of the translation start codon. Sequence analysis of the $1397 \mathrm{bp} 5^{\prime}$-flanking region using MOTIF-TRANSFAC 6.0 (Heinemeyer et al. 1999) revealed the presence of a TATA box at positions -33 to -19 relative to the

\footnotetext{
- 1321 CCTTAACCTG CACCACACTG TTGTTAGTTT TGGAgGTCAT TCATCCCTCG CTGGAgCTAA TTTGAGAGAC ATGTTTCTAA TTTTAGCGAA TTAGCAATAA - 1221 AACAAATATA TGCGTAAATC TCACTGGCCT CGGAAGCTCC CTCACTGAGC AACCCTGTGG CCTTCCATCT TGCTCTTATG CAATAGAAAA AGTTCAGAAC

-1121 AAGGTTAACA TCTGAGATTT TTAAACCCTC GCAGTAACTG AAAATCACAG ACATTATTGT GAGATGTGAT TTAAAAAAAT GTTTTGTTGA TGTTAATTAA USF

-1021 AAAAAAAAGA AAAAAAgGGA AAAATTGGGC TGAAAATTGT CTTTTTCAAG TGTTTGAAAg CAACTGAATT GAAGTGCAGA CAAAAGTGCA CAACAGAGTA -921 CCCAAAACGT ATCTAAAATA TGTCACAAAA TCGGAATGTG ATTAGAAAAT GTACATCAAA CCATTTGAAA TCTCACCATT ATAACTGAGG ATCCATAAAA - 821 CCTATAGTTC AAACTGTCAT ATATTGTGAA TAACTAAGAC ATGCATTGAA CTAATCAGCA TCTCTTACTT GATCATCTTC CTATTGTTCT TATAAACATG -721 CAACATTCTC ATATTGTCGA CCAAATTTTT ATTGTTGCCC TCTGGTCGAT GCTATAATGT GCCCAGGAGC AAATCTAATC GATATGCAAA GTCTTTTATT

-621 CCATCTGCCA TCAAACTCCT AAATTCAGAC CCCACTAATT TTAGAAGAAT TGAACCTATT TATTTATTTA CTCATTAATT TATTATTTAT GACGTACCCA USF

-521 TGTGCCTCTT AAATTGTTTT CTGACTGACT GAATGAATGA TCGTTGTAAT GATTTTATGT TTTTAATGTT TGATGTCTGG AAAAGCTACA ACTAAATTGC - 421 CCATTTGGGA TAAATAAAGT GGTCTGAACT GAACTGAGCT GAACATTCTC ATATTGTTCT TAGAGCCCTA TAAACAAACA GATTAGGCAT ATGAATATAT

-321 ACATTATATA AATCCATCCA AATGCTCAAT GATATCTGTA CATTTTGCTC CCTGCAATAA AAAACAACTG TTCTTAAAAT GTAATTCTTA CTTTGCAGTA

-221 AgTATGTCAg GTAGACTTGA ACTTTGgGtA TTCTTTGTGT GTGTGTGTGT GTGTTTGTGT GTGTGTATCT GTGTGTGTGT GGGTGGGTGT GTGTGTGCGG

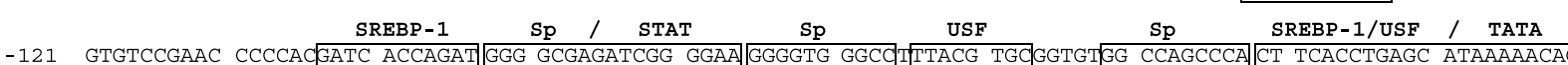
121 GIGTCCGAAC CCCCACGATC ACCAGAT GGG GCGAGATCGG GGAA GGGGTG GGCC ITACG TGC GGTGIGG CCAGCCCA CT TCACCIGAGC ATAAAAACAG

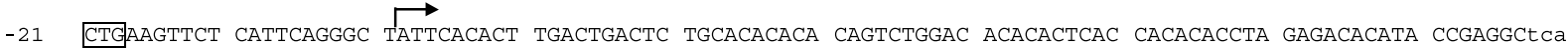
+80 tttagtcaaa cctgcggaga tg
}

Figure 1 Sequence analysis of the $5^{\prime}$-flanking region of $S$. aurata GCK. The upstream sequence isolated by chromosome walking is shown in capitals. An arrow indicates the transcription start site. The translation start codon is in bold and underlined. Putative binding sites for transcription factors are boxed. 
A

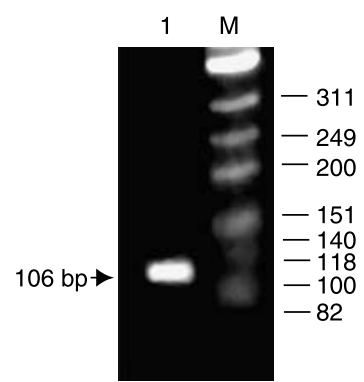

B

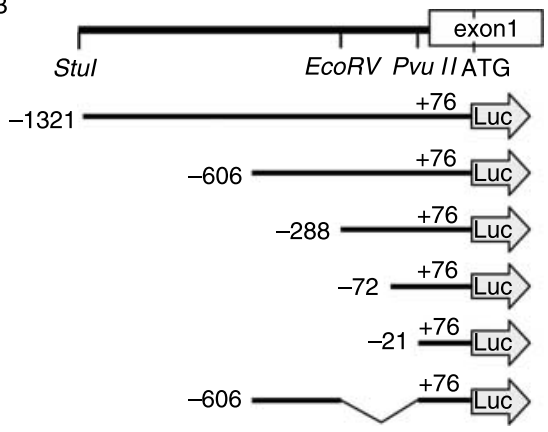

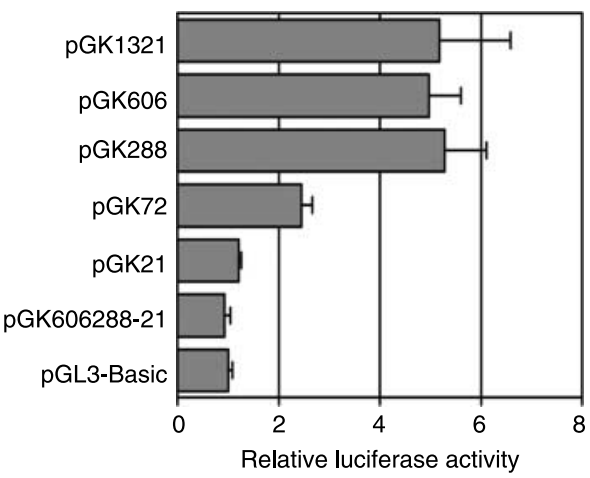

Figure 2 Mapping of the transcription initiation site of $S$. aurata GCK mRNA and functional analysis of the $5^{\prime}$-flanking region of GCK in HepG2 cells. (A) Resolution of SMART RACE PCR products on a 4\% agarose gel is shown. The assay, performed with $1 \mu \mathrm{g}$ hepatic Poly $\mathrm{A}^{+}$RNA (lane 1), produced a $106 \mathrm{bp}$ band that was purified, ligated into pGEM T easy and sequenced. M, DNA size marker ( $\phi$ X174 DNA digested with Hinfl). (B) The top left part represents the genomic organization of the $5^{\prime}$-flanking region of $S$. aurata GCK. Relevant restriction sites and exon 1, depicted as a white box, are indicated. Nucleotide numbering starts with +1 corresponding to the transcriptional start. Reporter constructs having varying $5^{\prime}$ ends and an identical $3^{\prime}$ end (+76) were transfected in HepG2 cells. Luciferase activity is expressed as a fold increase over promoterless reporter plasmid pGL3-Basic. Results presented are the mean \pm s.D. from at least three independent duplicate experiments.

transcription start site, and several putative transcription factor binding sites. Amongst the potential transcription factors were sites for Sp, USF, SREBP-1, and STAT proteins (Fig. 1). The nucleotide sequence reported in this paper was submitted to the DDBJ/ EMBL/GenBank databases under the accession number AY206500.

\section{Functionality of S. aurata GCK promoter}

To determine whether the genomic DNA flanking exon 1 contains a functional promoter, the DNA fragment isolated by chromosome walking was subcloned in the promoterless plasmid pGL3-Basic, upstream of the luciferase reporter gene. The recombinant plasmid pGK1321 ( - 1321/+76) was transiently co-transfected into HepG2 cells together with a lacZ-containing plasmid, as internal control for transfection efficiency. This construct exhibited more than a fivefold increase in luciferase activity relative to the promoterless vector, pGL3-Basic (Fig. 2B). This result indicated that the region comprised within 1321 nucleotides upstream of the transcription start site of $S$. aurata $G C K$ contains a functional promoter.

To examine the promoter functional regions involved in the modulation of basal $G C K$ expression in $S$. aurata, sequential $5^{\prime}$-deletion analysis of the promoter fragment was carried out. Deletion fragments, with $5^{\prime}$ ends ranging from -1321 to -21 and $3^{\prime}$ ends at +76 , were fused to the luciferase reporter gene and transfected into HepG2 cells. The longest $5^{\prime}$ construct (pGK1321, -1321 to +76 ) yielded a fivefold increase in luciferase activity relative to pGL3-Basic (Fig. 2B). Similar results were obtained using pGK606 $(-606$ to +76$)$ and pGK288 $(-288$ to +76$)$. The reporter construct containing the region -72 to +76 (pGK72) exhibited $\sim 50 \%$ of promoter activity compared with longer constructs. No activity was found using the smallest construct (pGK21; - 21 to +76 ) or a deleted construct harboring promoter sequences located between -606 and +76 bp relative to the transcription start site, but lacking the region comprised between positions -288 to -21 (pGK606 288-21). These results clearly indicate that the promoter region between -288 and $-21 \mathrm{bp}$ is important for the basal transcription of the $S$. aurata $G C K$ gene, suggesting that cis-acting elements may be located in this region. The high GC and GT content of this region led us to investigate a possible role of $\mathrm{Sp}$ proteins in the transcriptional activation of $G C K$.

\section{Transactivation of GCK promoter by Sp1 and repression of Sp1-mediated transcription by Sp3}

To examine the role of the putative Sp binding sites in the transcriptional activity of the $G C K$ gene, co-transfection experiments in HepG2 cells were performed. Reporter constructs containing $5^{\prime}$-deletion analysis of the promoter fragment were introduced into HepG2 cells together with expression plasmids encoding the Sp family members Sp1 or Sp3. Co-transfection of Sp1 with reporter constructs equal or longer than pGK72 resulted in a three- to fivefold induction of the promoter activity relative to the basal activity of the corresponding promoter constructs. No enhancement of the promoter activity could be detected when the shortest construct (pGK21) was used. These results suggest that a functional Sp binding site may be located within $72 \mathrm{bp}$ upstream of the transcription start site. Consistent with these results, no transactivation by Sp1 
was observed when co-transfected with pGK606 $\Delta 288-21$ reporter construct, which lacks the region between positions -288 and -21 (Fig. 3A). Sp3 alone did not affect the activity of any of the promoter fragments tested. However, co-transfection of a 1:1 mixture of expression vectors for $\mathrm{Sp} 1$ and $\mathrm{Sp} 3$ resulted in inhibition of the Sp1-dependent transactivation using pGK72 or longer reporter constructs. Since matches for Sp1 are found in the promoter sequences of mammalian GCK (Magnuson et al. 1989, Postic et al. 1995, Samson \& Wong 2002), we also analyzed the effect of Sp1 and Sp3 on the rat GCK promoter. To this end, we co-transfected HepG2 cells with expression vectors for Sp1 and Sp3, and a reporter construct harboring a promoter sequence of rat GCK (prGK1418; positions $-1418 \mathrm{bp}$ to $+109 \mathrm{bp}$ relative to the transcription start site) that includes a putative Sp binding site (positions -441 to -433 ; Magnuson et al. 1989). As in $S$. aurata, Sp1 transactivated rat $G C K$ promoter and Sp3 repressed Sp1-mediated transcription (Fig. 3A).

As Sp1 and Sp3 behaved functionally different and can compete for the same binding site, we investigated the potential function of Sp3 as a repressor of S. aurata
GCK promoter. Various amounts $(0 \cdot 8-3 \cdot 2 \mu \mathrm{g})$ of the expression vectors encoding Sp1 and Sp3 were co-transfected together with the pGK72 reporter construct into HepG2 cells. As shown in Fig. 3B, the promoter activity decreased in a dose-dependent manner when the cells were co-transfected with $0 \cdot 8 \mu \mathrm{g}$ pRC-CMVSp1 and increasing amounts of pRCCMVSp3. However, co-transfection with $0 \cdot 8 \mu \mathrm{g}$ of pRCCMVSp3 and increasing amounts of pRC-CMVSp1 resulted in a dose-dependent increase of promoter transcriptional activity. These results suggest that Sp1 and Sp3 compete for the same binding site in the GCK promoter.

\section{Sp1 and Sp3 bind to the putative Sp binding site of GCK promoter}

The shorter reporter construct that exhibited Sp1mediated transactivation (pGK72) contains a GC-rich region at positions -53 to -44 relative to the transcription start site of $S$. aurata GCK. To show that Sp1 and Sp3 can indeed bind to the putative Sp binding site at position -53 to -44 , bandshift experiments were performed. Using probe GK-56/-37 (harboring
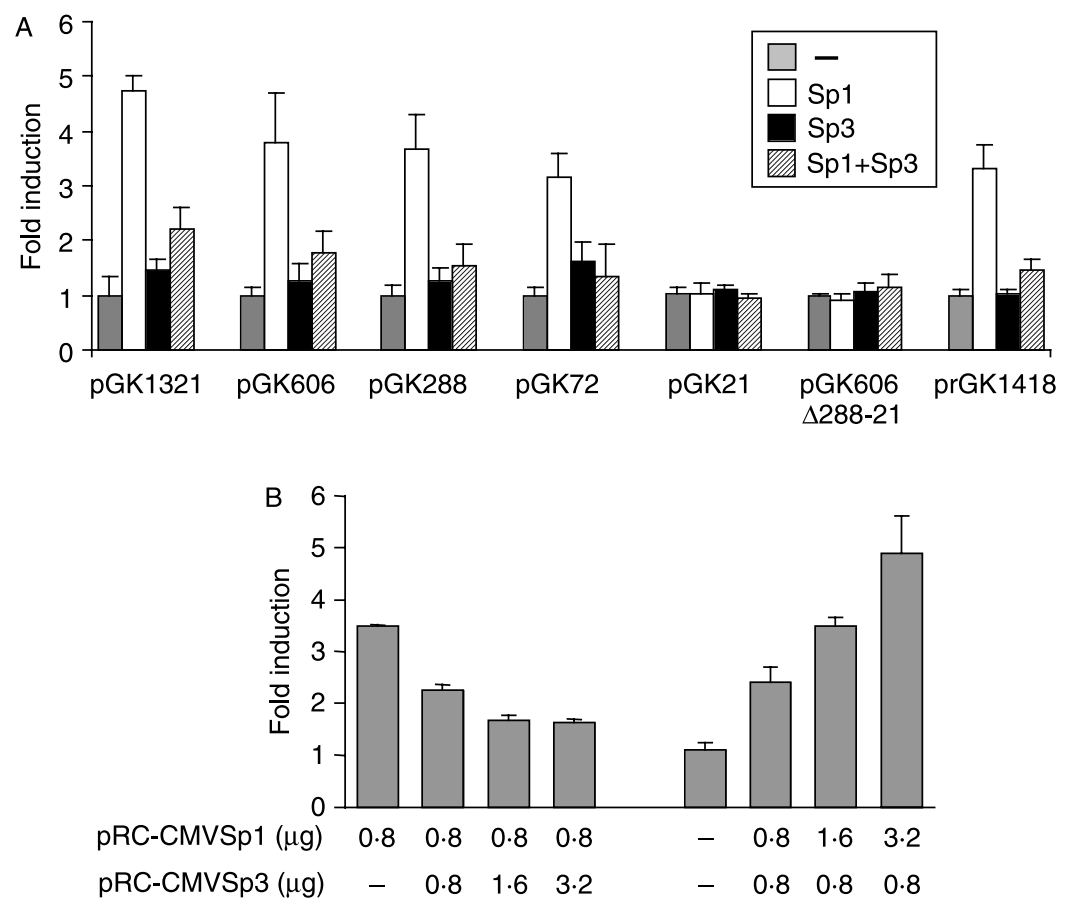

Figure 3 Effect of Sp1 and Sp3 on S. aurata GCK promoter transcription in HepG2 cells. (A) The cells were transfected with pGL3-Basic, the $S$. aurata GCK promoter constructs pGK1321, pGK606, pGK288, pGK72, pGK21, or pGK606 288-21, or the rat GCK promoter construct prGK1418, with or without expression plasmids encoding Sp1 or Sp3. (B) The cells were co-transfected with pGK72 and different amounts of pRCCMV Sp1 and/or pRC-CMV Sp3. The promoter activity of the constructs alone was set at 1 . The data represent the mean \pm s.D. values of two independent duplicate experiments. 
the putative Sp binding site) and HepG2 extracts prepared from cells overexpressing Sp1, one major shifted band could be observed. A DNA-protein complex with the same mobility was observed when a consensus Sp1 probe was used (Sp1-cons). The shifted band was eliminated by competition with 200-fold molar excess of unlabeled Sp1-cons (Fig. 4A). Similar results were obtained using HepG2 extracts prepared from Sp3 overexpressing cells (Fig. 4B). Specificity of Sp1 and Sp3 binding to the Sp box at positions -53 to -44 of $S$. aurata $G C K$ was validated through supershift experiments using the labeled GK-56/-37 probe and in vitro translated Sp1 and Sp3. Sp1 and Sp3 gave rise to complexes of similar mobility. In the presence of Sp1 antibody, the complex observed with Spl protein was partially supershifted. Similar results were obtained using a Sp3 antibody in the presence of Sp3 protein. A supershifted band of less mobility was observed when both anti-Sp1 and anti-Sp3 antibodies were included in a single reaction with Sp1 and Sp3 proteins (Fig. 4C). Altogether, these data confirm that both Sp1 and Sp3 bind the $\mathrm{Sp}$ box at positions -53 to -44 of GCK.

To test whether we could abolish formation of the DNA-protein complex by mutating the putative Sp binding site, we performed bandshift assays using HepG2 extracts of Sp1 overexpressing cells and a labeled probe harboring positions -56 to -37 of the GCK gene in which the GC box was mutated (GK-56/37 mutSp). As shown in Fig. $4 \mathrm{D}$, the complex shifted using Sp1-cons and GK-56/-37 probes totally disappeared when the GK-56/-37mutSp probe was used.

\section{Mutating the Sp binding site abolishes transactivation by $\mathrm{Sp} 1$}

The same mutations as described for the doublestranded oligonucleotide GK-56/-37mutSp used in bandshift assays were introduced into the promoter constructs pGK288 and pGK72 to generate reporter constructs with a mutated Sp site (pGK288mutSp and pGK72mutSp respectively). Co-transfection experiments in HepG2 cells with the promoter constructs and expression vectors for Sp1 and Sp3 were performed to compare transactivation of the wild type and mutated reporter constructs. Sp1 was not able to enhance the transcription of the constructs harboring the mutated Sp site (Fig. 5A). From these studies, we concluded that the Sp binding site at positions -53 to -44 , relative to the major transcription start site of the $S$. aurata $G C K$ promoter, is responsible for transactivation by Sp1. In addition, Sp3 is a repressor of Sp1-mediated activation by competing for the same binding site.

The activating effect of $\mathrm{Sp} 1$ on $G C K$ transcription was also tested in nonliver-derived cells. Transient transfection of CHO and SBL cells with an expression plasmid encoding Sp1 and the $S$. aurata GCK reporter constructs
pGK72 and pGK72mutSp showed that the effect of Sp1 was dependent on the cell line. Similarly, as in HepG2 cells, Sp1 activated GCK transcription in CHO cells and did not transactivate a $G C K$ reporter construct harboring a mutated $\mathrm{Sp}$ site at positions -53 to -44 . In contrast to HepG2 and CHO cells, no effect of Sp1 was observed in SBL cells (Fig. 5B).

\section{Regulation of Sp1 protein levels and GCK expression by insulin in liver of $S$. aurata}

Although Sp1 binding sites are common to many insulin-responsive genes and matches for Sp1 are found in the promoter sequences of rat and mouse GCK (Magnuson et al. 1989, Postic et al. 1995, Samson \& Wong 2002), the putative involvement of Sp proteins in transactivation of GCK has not been studied. To study the action of insulin and the role of Sp1 and Sp3 on $G C K$ expression, we analyzed GCK mRNA abundance and protein levels of Sp1 and Sp3 in the liver of insulintreated $S$. aurata. Analysis by northern blot revealed that GCK mRNA increased after insulin treatment in the piscine liver (Fig. 6A). Western blot analysis showed that insulin increased Sp1 protein levels, whereas no effect on Sp3 was observed (Fig. 6B).

\section{Discussion}

It is well known that insulin stimulates $G C K$ expression in the liver. However, knowledge of the transcription factors implicated in control of $G C K$ transcription has received limited attention and the molecular mechanism responsible for this effect remains unclear. In this study, we have isolated and characterized the promoter region of $G C K$ from $S$. aurata. The genomic fragment isolated allowed us to identify a cis-element in the proximal region of the GCK promoter implicated in transactivation by Sp1. Additionally, we present evidence that supports a role for Sp proteins in insulindependent activation of $G C K$ by insulin.

The $5^{\prime}$-flanking region of $S$. aurata $G C K$ was cloned by chromosome walking. Mapping of the transcriptional start indicated the presence of a start site located $98 \mathrm{bp}$ upstream relative to the translation start codon. Our localization of the transcription start site predicts a $2058 \mathrm{bp}$ mRNA, which is consistent with the $2 \cdot 1 \mathrm{~kb}$ transcript previously identified by northern blot (Caseras et al. 2000). The functionality of the putative promoter region of fish $G C K$ was tested by transient transfection of HepG2 cells with fusion constructs of the $1397 \mathrm{bp}$ isolated by chromosome walking and sequential $5^{\prime}$ deletions of this fragment to the luciferase gene. We found that the promoter region within $288 \mathrm{bp}$ upstream to the transcription start site is essential for transcriptional basal activity of $G C K$ and thus 
A

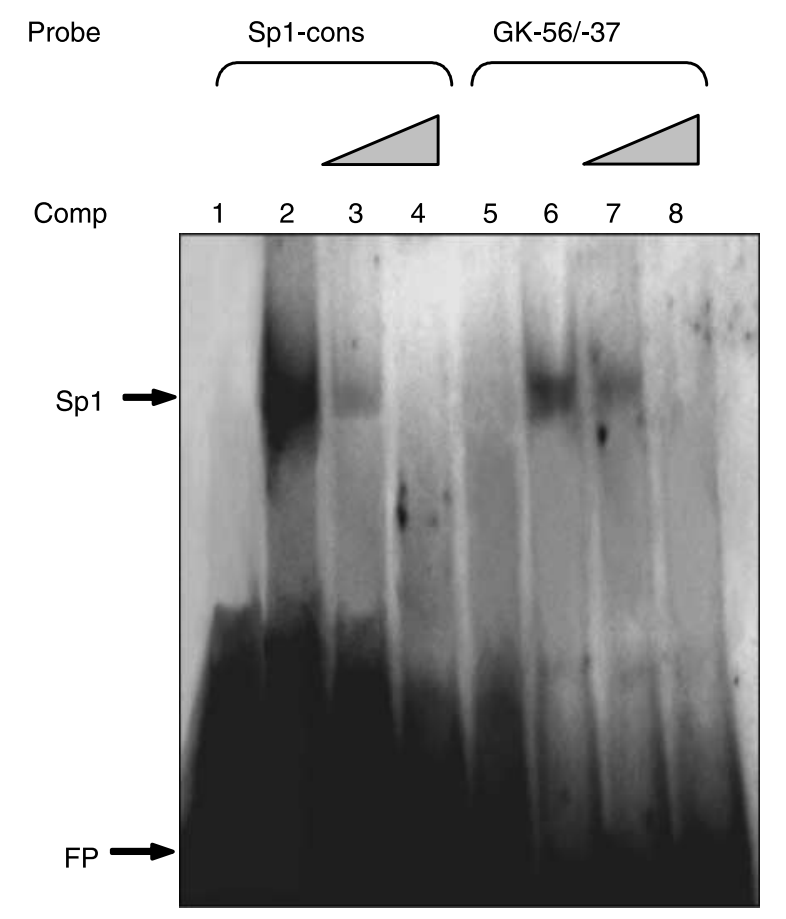

c

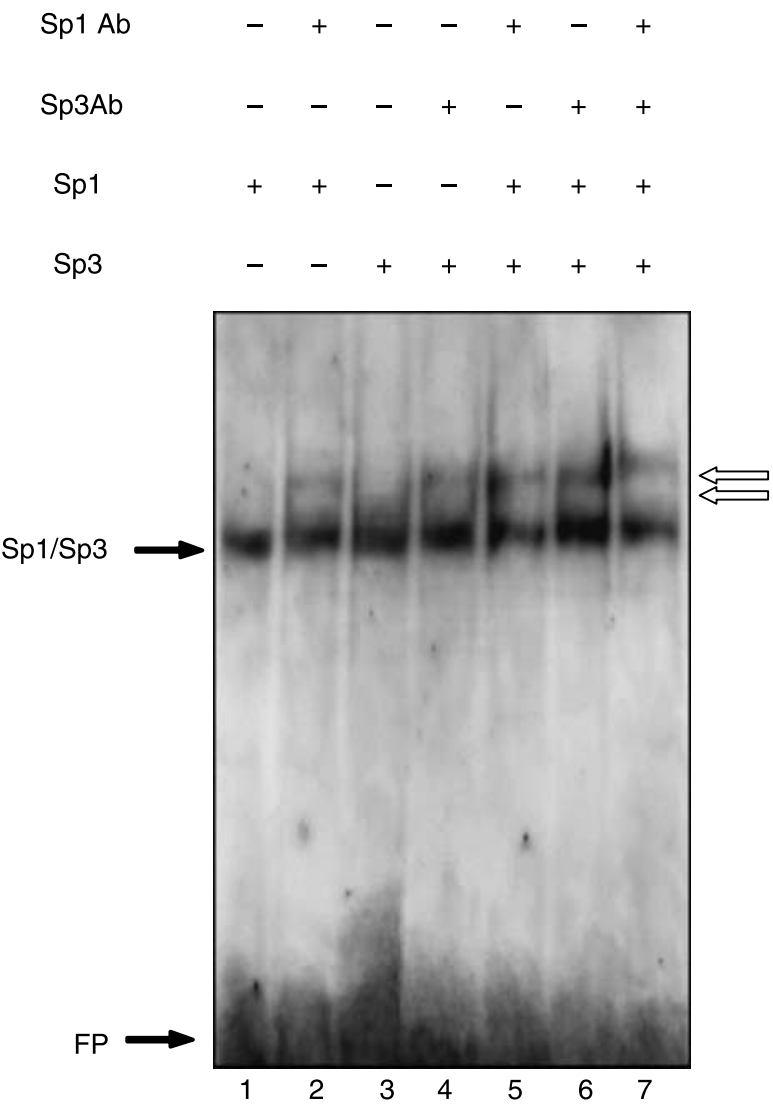

B

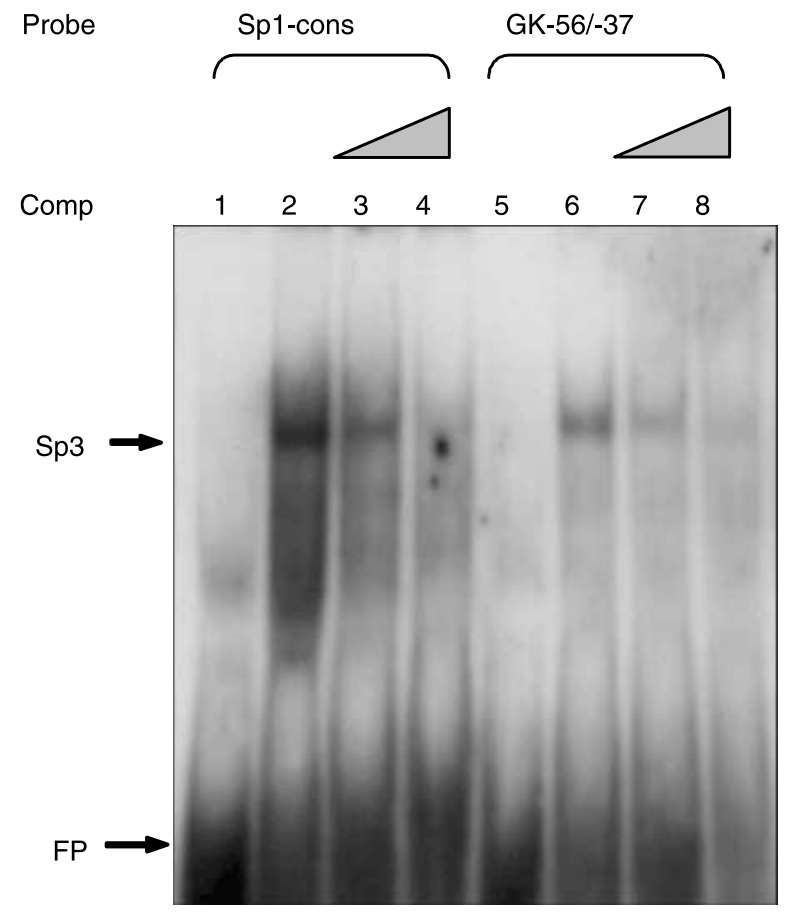

D

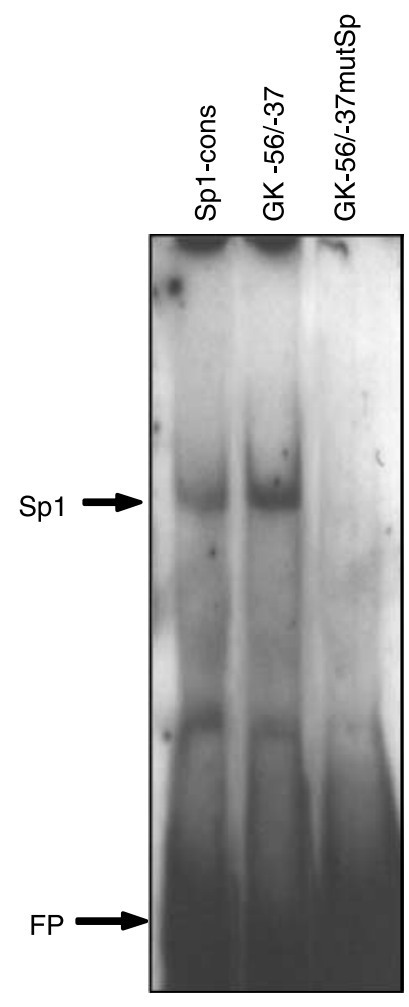



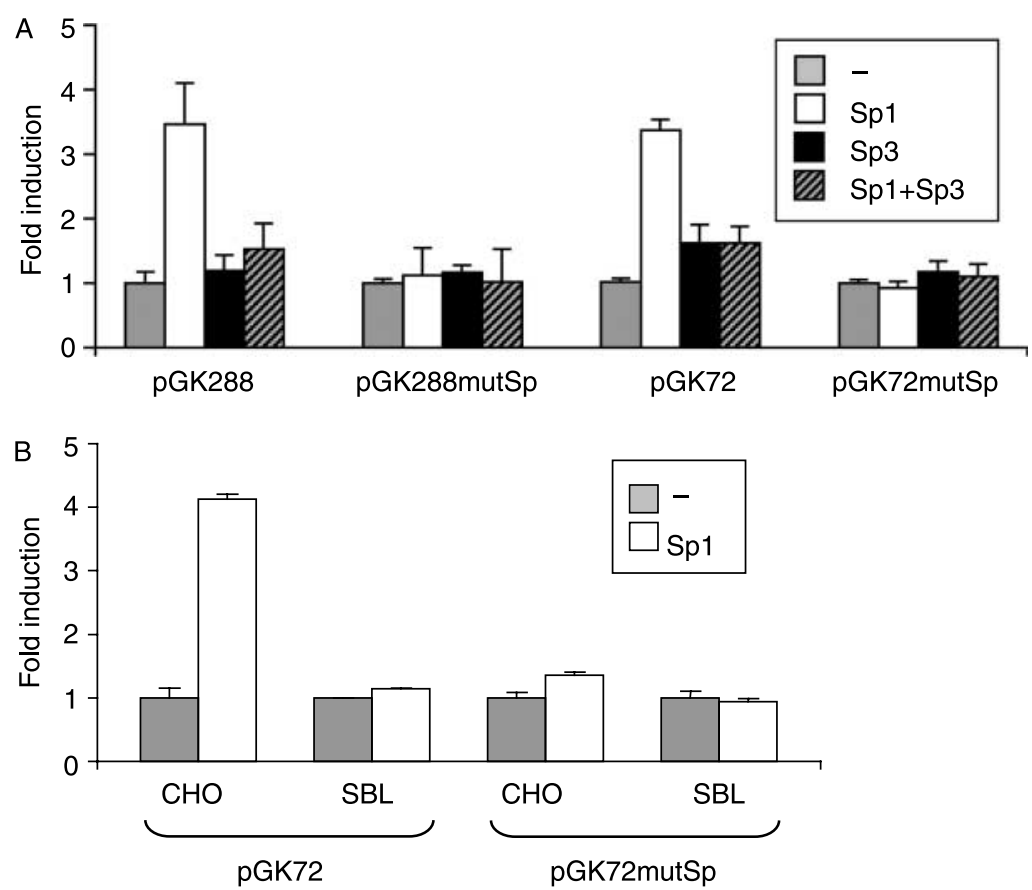

Figure 5 Effect of Sp1 and Sp3 on the promoter activity of GCK containing a mutated Sp box. (A) pGK288, pGK288mutSp, pGK72, and pGK72mutSp were co-transfected in HepG2 cells and with expression vectors encoding Sp1 and/or Sp3. (B) CHO and SBL cells were transfected with pGK72 and pGK72mutSp, and with or without an expression plasmid encoding Sp1. The luciferase activity of the reporter constructs alone was set at 1 . The data represent the mean \pm s.D. values of two independent duplicate experiments.

constitutes the core functional promoter of this gene. The high GC and GT content of this region led us to investigate a possible role of $\mathrm{Sp}$ proteins in the transcriptional activation of $G C K$.

GC and GT boxes are DNA-elements frequently present in many promoters and enhancers of genes that are under cell cycle regulation, hormonal activation, and developmental patterning. The Sp family of proteins comprises a large number of homologous transcription factors that can control transcriptional activity of target genes through binding to GC/GT boxes in their regulatory regions (Suske 1999, Bouwman \& Philipsen 2002, Kaczynski et al. 2003). Sp proteins have been found in species ranging from invertebrates to humans, including fish (Baudler et al. 1997, Zhao et al. 2003). Sp family members contain three zinc fingers and glutamine- and serine/ threonine-rich trans-activation domains. Despite the high degree of structural homology, Sp proteins behave in a functionally different manner. Regulation of transcription via GC/GT boxes by $\mathrm{Sp}$ proteins is a tightly controlled complex process. Sp1 and Sp3 recognize and bind to the GC/GT boxes with identical affinity (Hagen et al. 1994). Sp1 is implicated in the activation of a large number of regulated and constitutively expressed genes, whereas Sp3 comprises an additional inhibitory domain and acts as an activator or as a repressor of Sp1-mediated activation depending on the promoter and cellular context (Suske 1999, Bouwman \& Philipsen 2002, Kaczynski et al. 2003). Since Sp1 and Sp3 are present in the same cell types and compete for the same binding sites in vivo, the relative abundance of both transcription factors allows regulation of target genes. In the present study, we show

Figure 4 Electrophoretic mobility shift assay. Competition analysis using (A) Sp1 or (B) Sp3 overexpressing HepG2 extracts incubated with labeled oligonucleotides Sp1-cons (lanes 1-4) or GK-56/-37 (lanes 5-8). Lanes 1 and 5 contained no extract. Lanes 2 and 6 show binding of HepG2 extracts to labeled probes without competitor. Lanes 3, 7 and 4, 8 show competition with 10- and 200-fold molar excess respectively of unlabeled double-stranded competitor (Sp1-cons). (C) Gel shift with labeled probe GK-56/-37 using in vitro translated Sp1 (Sp1; lanes 1, 2, 5-7) and/or Sp3 (Sp3; lanes 3-7), and supershift performed with antibodies against Sp1 (Sp1 Ab; lanes 2, 5 and 7) and/or Sp3 (Sp3 Ab; lanes 4, 6, and 7). (D) Shift assays performed using HepG2 extracts incubated with labeled oligonucleotides Sp1-cons, GK56/-37 or GK-56/-37mutSp (mutated Sp1 binding sequence). DNA-protein complexes and supershifted bands are indicated by arrows and open arrows respectively. FP, free probes. 


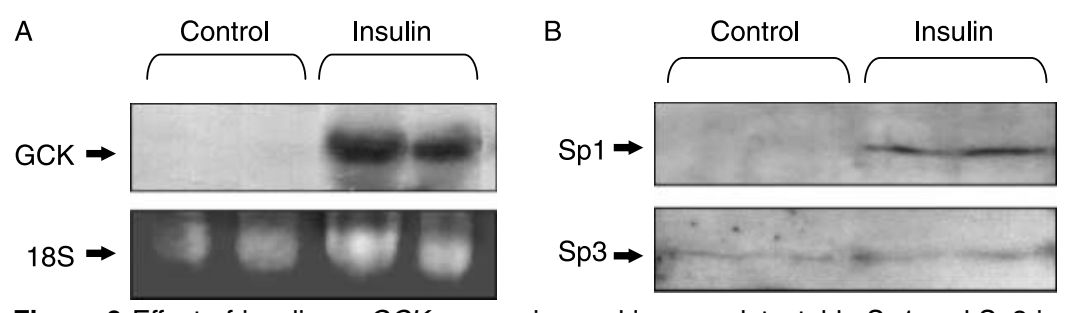

Figure 6 Effect of insulin on GCK expression and immunodetectable Sp1 and Sp3 in the liver of $S$. aurata. (A) Representative northern blot of GCK mRNA levels in liver of fish after $6 \mathrm{~h}$ treatment with saline (control) or insulin (10 U/kg fish). The integrity and relative amounts of RNA loaded were checked and corrected by $18 \mathrm{~S}$ rRNA ethidium bromide staining on the same gel. (B) Western blotting analysis of Sp1 and Sp3 protein levels in liver of fish after $6 \mathrm{~h}$ treatment with saline (control) or insulin (10 U/kg fish).

that both Sp1 and Sp3 are capable of interacting specifically with the GC box located between $-53 \mathrm{bp}$ and -44 bp upstream of the transcription start site of GCK.

To determine the functional role of Sp1 and Sp3 on the GCK promoter, transient transfection studies were carried out in HepG2 cells. We demonstrate that Sp1 confers an activating signal through binding to the $G C K$ promoter. Mutations in the GC box abolish binding of Sp1 to the mutated site. As a result Sp1 was no longer able to enhance the transcription of $G C K$ in transient transfection experiments. These results clearly indicate that $\mathrm{Sp} 1$ is implicated in the transcriptional activation of GCK promoter. On the other hand, Sp3 did not drive reporter gene expression, and furthermore it behaved as a repressor of Sp1-mediated transactivation by competing for the same binding site in the GCK promoter. Interestingly, transfection experiments performed with a promoter reporter construct of rat GCK harboring a putative Sp binding site gave rise to similar results as with $S$. aurata $G C K$, suggesting that regulation of GCK transcription by Sp proteins may not be a specific effect for fish species. On the other hand, an elevated Sp1 to Sp3 ratio resulted in stimulation of the $S$. aurata GCK promoter activity, whereas the gene expression was inhibited when the Sp3 to Sp1 ratio was high. In support of this model, it has been shown that a mutant of Sp3 lacking the DNA-binding domain is not able to suppress Spl-mediated transactivation of the artificial promoter BCAT-2 (Hagen et al. 1994). In the present study, most of the transfection experiments were performed in a liver-derived cell line (HepG2). However, we also analyzed whether the activating effect of Sp1 on S. aurata GCK is cell type-dependent. As in HepG2 cells, Sp1 transactivated GCK transcription in CHO cells, whereas it did not cause any effect on SBL cells. Dependence of the cell lines to Sp1 action may result from cell-type dependent features such as posttranslational regulation of $\mathrm{Sp} 1$ and the $\mathrm{Sp} 1 / \mathrm{Sp} 3$ ratio, which in turn may confer specificity of $G C K$ tissue expression.
In addition to the steady-state levels of Sp1, hormones can alter its binding and transcriptional activity through post-translational modifications. Insulin stimulates synthesis, O-glycosylation and phosphorylation of Sp1 (Bouwman \& Philipsen 2002, Samson \& Wong 2002, Chu \& Ferro 2005, Majumdar et al. 2006). The fact that Sp1 binding sites are found in the insulin-responsive regions of numerous genes suggests that Sp1 is involved in mediating insulin action. In particular, Spl plays an important role in the transcription of several glycolytic enzymes, such as pyruvate kinase and aldolase (Hermfisse et al. 1996, Schafer et al. 1996, Netzker et al. 1997). Here, we found that insulin increased GCK mRNA levels in the liver of $S$. aurata. These results are consistent with previous studies in $S$. aurata that indicate decreased GCK mRNA in starved fish, postprandial rise of GCK expression, and increased GCK mRNA abundance in liver of fish fed high carbohydrate diets (Caseras et al. 2000, 2002). Indeed, we recently showed that insulin stimulates the expression of 6-phosphofructo-2-kinase/fructose-2,6bisphosphatase and down-regulates the gluconeogenic glucose-6-phosphatase catalytic subunit in the liver of this species (Salgado et al. 2004, Metón et al. 2006). In addition to stimulation of hepatic GCK expression, we found that insulin increased $\mathrm{Sp} 1$ protein levels in $S$. aurata, whereas Sp3 remained unaffected. These results suggest that increased Sp1/Sp3 ratio in insulin-treated animals may enhance $G C K$ transcription.

In conclusion, the present study has shown that $\mathrm{Sp} 1$ binds to and transactivates $G C K$ promoter, and points to involvement of Sp proteins in insulin regulation of $G C K$ expression.

\section{Acknowledgements}

The authors thank Dr G Suske for providing the Sp1 and Sp3 expression vectors, and Dr J Castric for supplying the SBL cell line. This work was supported by the MCYT (Spain) grants BMC2000-0761 and BIO2003-01098. We thank the Language Advisory Service of the University of Barcelona for correcting 
the English manuscript. The authors declare that there is no conflict of interest that would prejudice the impartiality of this scientific work.

\section{References}

Baudler M, Duschl J, Winkler C, Schartl M \& Altschmied J 1997 Activation of transcription of the melanoma inducing Xmrk oncogene by a GC box element. Journal of Biological Chemistry 272 131-137.

Bouwman P \& Philipsen S 2002 Regulation of the activity of Sp1related transcription factors. Molecular and Cellular Endocrinology 195 27-38.

Caseras A, Metón I, Fernández F \& Baanante IV 2000 Glucokinase gene expression is nutritionally regulated in liver of gilthead sea bream (Sparus aurata). BBA - Gene Structure and Expression 1493 135-141.

Caseras A, Metón I, Vives C, Egea M, Fernández F \& Baanante IV 2002 Nutritional regulation of glucose-6-phosphatase gene expression in liver of the gilthead sea bream (Sparus aurata). British Journal of Nutrition 88 607-614.

Cha JY, Kim HI, Im SS, Li TZ \& Ahn YH 2001 HNF1 and/or HNF3 may contribute to the tissue specific expression of glucokinase gene. Experimental and Molecular Medicine 33 59-63.

Chauhan J \& Dakshinamurti K 1991 Transcriptional regulation of the glucokinase gene by biotin in starved rats. Journal of Biological Chemistry 266 10035-10038.

Christiansen DC \& Klungsøyr L 1987 Metabolic utilization of nutrients and the effects of insulin in fish. Comparative Biochemistry and Physiology 88B 701-711.

Chu S \& Ferro TJ 2005 Sp1: regulation of gene expression by phosphorylation. Gene 348 1-11.

Cowey CB \& Walton MJ 1989 Intermediary metabolism. In Fish Nutrition, pp 260-321. Ed JE Halver. San Diego: Academic Press.

Foretz M, Guichard C, Ferre P \& Foufelle F 1999 Sterol regulatory element binding protein-1c is a major mediator of insulin action on the hepatic expression of glucokinase and lipogenesis-related genes. PNAS 96 12737-12742.

Graham FL \& Van der Eb AJ 1973 A new technique for assay of infectivity of human adenovirus 5 DNA. Virology $\mathbf{5 2} 456-467$.

Gregori C, Guillet-Deniau I, Girard J, Decaux JF \& Pichard AL 2006 Insulin regulation of glucokinase gene expression: evidence against a role for sterol regulatory element binding protein 1 in primary hepatocytes. FEBS Letters 580 410-414.

Hagen G, Müller S, Beato M \& Suske G 1994 Spl-mediated transcriptional activation is repressed by Sp3. EMBO Journal 13 3843-3851.

Hansmannel F, Mordier S \& Iynedjian PB 2006 Insulin induction of glucokinase and fatty acid synthase in hepatocytes: analysis of the roles of sterol-regulatory-element-binding protein-1c and liver $\mathrm{X}$ receptor. Biochemical Journal 399 275-283.

Heinemeyer T, Chen X, Karas H, Kel AE, Kel OV, Liebich I, Meinhardt T, Reuter I, Schacherer F \& Wingender E 1999 Expanding the TRANSFAC database towards an expert system of regulatory molecular mechanisms. Nucleic Acids Research 27 318-322.

Hermfisse U, Schafer D, Netzker R \& Brand K 1996 The aldolase A promoter in proliferating rat thymocytes is regulated by a cluster of SP1 sites and a distal modulator. Biochemical and Biophysical Research Communications 225 997-1005.

Iynedjian PB 1993 Mammalian glucokinase and its gene. Biochemical Journal 293 1-13.

Iynedjian PB 1998 Identification of upstream stimulatory factor as transcriptional activator of the liver promoter of the glucokinase gene. Biochemical Journal 333 705-712.
Iynedjian PB, Jotterand D, Nouspikel T, Asfari M \& Pilot PR 1989 Transcriptional induction of glucokinase gene by insulin in cultured liver cells and its repression by the glucagon-cAMP system. Journal of Biological Chemistry 264 21824-21829.

Iynedjian PB, Marie S, Wang H, Gjinovci A \& Nazaryan K 1996 Liverspecific enhancer of the glucokinase gene. Journal of Biological Chemistry 271 29113-29120.

Kaczynski J, Cook T \& Urrutia R 2003 Sp1- and Kruppel-like transcription factors. Genome Biology 4206.

Kim SY, Kim HI, Park SK, Im SS, Li T, Cheon HG \& Ahn YH 2004 Liver glucokinase can be activated by peroxisome proliferator-activated receptor-gamma. Diabetes 53 S66-S70.

Lannoy VJ, Decaux JF, Pierreux CE, Lemaigre FP \& Rousseau GG 2002 Liver glucokinase gene expression is controlled by the onecut transcription factor hepatocyte nuclear factor-6. Diabetologia $\mathbf{4 5}$ $1136-1141$

Magnuson MA 1990 Glucokinase gene structure. Diabetes 39 523-527.

Magnuson MA \& Shelton KD 1989 An alternate promoter in the glucokinase gene is active in the pancreatic beta cell. Journal of Biological Chemistry 264 15936-15942.

Magnuson MA, Andreone TL, Printz RL, Koch S \& Granner DK 1989 Rat glucokinase gene: structure and regulation by insulin. PNAS $\mathbf{8 6}$ 4838-4842.

Majumdar G, Harrington A, Hungerford J, Martinez-Hernandez A, Gerling IC, Raghow R \& Solomon S 2006 Insulin dynamically regulates calmodulin gene expression by sequential $O$-glycosylation and phosphorylation of $\mathrm{Spl}$ and its subcellular compartmentalization in liver cells. Journal of Biological Chemistry 281 3642-3650.

Metón I, Egea M \& Baanante IV 2003 New insights into the regulation of hepatic glucose metabolism in fish. In Recent Research Developments in Biochemistry, vol 4, pp 125-149. Ed SG Pandalai. Kerala, India: Research Signpost

Metón I, Egea M, Anemaet IG, Fernández F \& Baanante IV 2006 Sterol regulatory element binding protein-1a transactivates 6-phosphofructo-2-kinase/fructose-2,6-bisphosphatase gene promoter. Endocrinology 147 3446-3456.

Moon TW 2001 Glucose intolerance in teleost fish: fact or fiction? Comparative Biochemistry and Physiology 29B 243-249.

Narkewicz MR, Iynedjian PB, Ferre P \& Girard J 1990 Insulin and triiodothyronine induce glucokinase mRNA in primary cultures of neonatal rat hepatocytes. Biochemical Journal 271 585-589.

Netzker R, Weigert C \& Brand K 1997 Role of the stimulatory proteins Sp1 and Sp3 in the regulation of transcription of the rat pyruvate kinase M gene. European Journal of Biochemistry $\mathbf{2 4 5}$ $174-181$.

Postic C, Niswender KD, Decaux JF, Parsa R, Shelton KD, Gouhot B, Pettepher CC, Granner DK, Girard J \& Magnuson MA 1995 Cloning and characterization of the mouse glucokinase gene locus and identification of distal liver-specific DNase I hypersensitive sites. Genomics 29 740-750.

Ribaux P, Gjinovci A, Sadowski HB \& Iynedjian PB 2002 Discrimination between signaling pathways in regulation of specific gene expression by insulin and growth hormone in hepatocytes. Endocrinology 143 3766-3772.

Roth U, Jungermann K \& Kietzmann T 2002 Activation of glucokinase gene expression by hepatic nuclear factor 4alpha in primary hepatocytes. Biochemical Journal 365 223-228.

Roth U, Curth K, Unterman TG \& Kietzmann T 2004 The transcription factors HIF-1 and HNF-4 and the coactivator p300 are involved in insulin-regulated glucokinase gene expression via the phosphatidylinositol 3-kinase/protein kinase B pathway. Journal of Biological Chemistry 279 2623-2631.

Salgado MC, Meton I, Egea M \& Baanante IV 2004 Transcriptional regulation of glucose-6-phosphatase catalytic subunit promoter by insulin and glucose in the carnivorous fish, Sparus aurata. Journal of Molecular Endocrinology 33 783-795. 
Samson SL \& Wong NC 2002 Role of Sp1 in insulin regulation of gene expression. Journal of Molecular Endocrinology 29 265-279.

Sawka-Verhelle D, Tartare-Deckert S, Decaux JF, Girard J \& Van Obberghen E 2000 Stat 5B, activated by insulin in a Jakindependent fashion, plays a role in glucokinase gene transcription. Endocrinology 141 1977-1988.

Schafer D, Hamm-Kunzelmann B, Hermfisse U \& Brand K 1996 Differences in DNA-binding efficiency of Sp1 to aldolase and pyruvate kinase promoter correlate with altered redox states in resting and proliferating rat thymocytes. FEBS Letters 391 35-38.

Suske G 1999 The Sp-family of transcription factors. Gene 238 291-300.

Wilson RP 1994 Utilization of dietary carbohydrate by fish. Aquaculture $12467-80$.
Zhang W, Patil S, Chauhan B, Guo S, Powell DR, Le J, Klotsas A, Matika R, Xiao X, Franks R et al. 2006 FoxO1 regulates multiple metabolic pathways in the liver: effects on gluconeogenic, glycolytic, and lipogenic gene expression. Journal of Biological Chemistry 281 10105-10117.

Zhao J, Cao Y, Zhao C, Postlethwait J \& Meng A 2003 An SP1-like transcription factor Spr2 acts downstream of Fgf signaling to mediate mesoderm induction. EMBO Journal 22 6078-6088.

Received in final form 2 February 2007 Accepted 13 February 2007

Made available online as an Accepted Preprint 14 February 2007 